\title{
Evaluation of sealed containers for use in centrifuges by a dynamic microbiological test method
}

\author{
GJ HARPER
}

From the Environmental Microbiology and Safety Reference Laboratory, Public Health Laboratory Service, Centre for Applied Microbiology and Research, Porton Down, Salisbury, Wiltshire

SUMMARY A dynamic microbiological test has been used to assess the safety of sealed containers for use in laboratory centrifuges. When 26 models of containers (buckets or rotors) were examined $27 \%$ failed to contain aerosols. Some of the reasons for the failures are described.

Sealed buckets and rotors are supplied by most manufacturers of laboratory centrifuges to protect users from exposure to aerosols that can arise from spillage or breakage of tubes or bottles during centrifugation.

In the recently revised British Standard BS4402' Appendix D describes methods for assessing the safety of sealed buckets and rotors using a solution of sodium fluorescein. Sealed buckets are tested by measuring leakage when the buckets are held in an inverted position over white filter paper in a vacuum chamber at a negative pressure of less than $4 \mathrm{kPa}$ ( $30 \mathrm{~mm} \mathrm{Hg}$ ) for $3 \mathrm{~min}$, with the buckets containing enough solution to cover the seal between cap and bucket. The paper is examined in a darkened room using ultraviolet light at a wavelength of $365 \mathrm{~nm}$. If no fluorescence is seen on the filter paper, or in washings from the exterior of the seal, the buckets are deemed to have passed the test for leakage. For sealed rotors containers are over filled with solution and the rotor centrifuged at its highest speed for 10 min. Strips of filter paper attached vertically inside the centrifuge chamber to surfaces immediately surrounding the rotor are examined for fluorescence in the same way as for sealed buckets.

Although these tests using fluorescein are rapid and convenient, they suffer from two shortcomings: (a) tests using sodium fluorescein are not as sensitive as a microbiological method and will fail to detect the smaller airborne particles, which are the most hazardous for infection by inhalation ${ }^{2}$; and $(b)$ the test for sealed buckets does not mimic what happens when a bottle or tube leaks or breaks when it is being rotated at high speed.

To simulate the worst in use situation, breakage of

Accepted for publication 4 July 1984 a bottle or tube inside a sealed container during centrifugation, a dynamic test using bacterial spores, applicable to both sealed buckets and rotors, has been used.

\section{Material and methods}

\section{TEST BOTTLES}

Standard one ounce glass screw-capped bottles were deliberately cracked by heating in a flame and plunging into cold water. This produced multiple cracks (Fig. 1). After cracking, bottles were tested for gross leakage by filling with water and allowing to stand on a sheet of filter paper for several minutes. Only bottles showing no gross leaks were used for tests. To ensure breakage during centrifugation a small ball bearing was placed between the base of the bottle and the bottom of the centrifuge bucket (Fig. 2).

\section{TEST SUSPENSION}

An aqueous suspension of spores of Bacillus subtilis var globigii NCTC no 10073 containing $1 \times 10^{10}$ viable spores per millilitre was used.

\section{TEST METHOD}

Cracked bottles containing $20 \mathrm{ml}$ of suspension and closed with screw caps were placed in the buckets or rotor being tested and centrifuged for $8 \mathrm{~min}$ at the maximum recommended speed for the equipment. The centrifuge was contained in a sealed chamber with a high efficiency particulate air filter on the air inlet. ${ }^{3}$ Air was extracted from the test chamber by means of air sampling equipment placed close to the centrifuge (see below) and surfaces in and around the centrifuge were sampled using sterile cotton wool swabs moistened with sterile water. Control samples of air and from surfaces were collected before each test to measure any background con- 


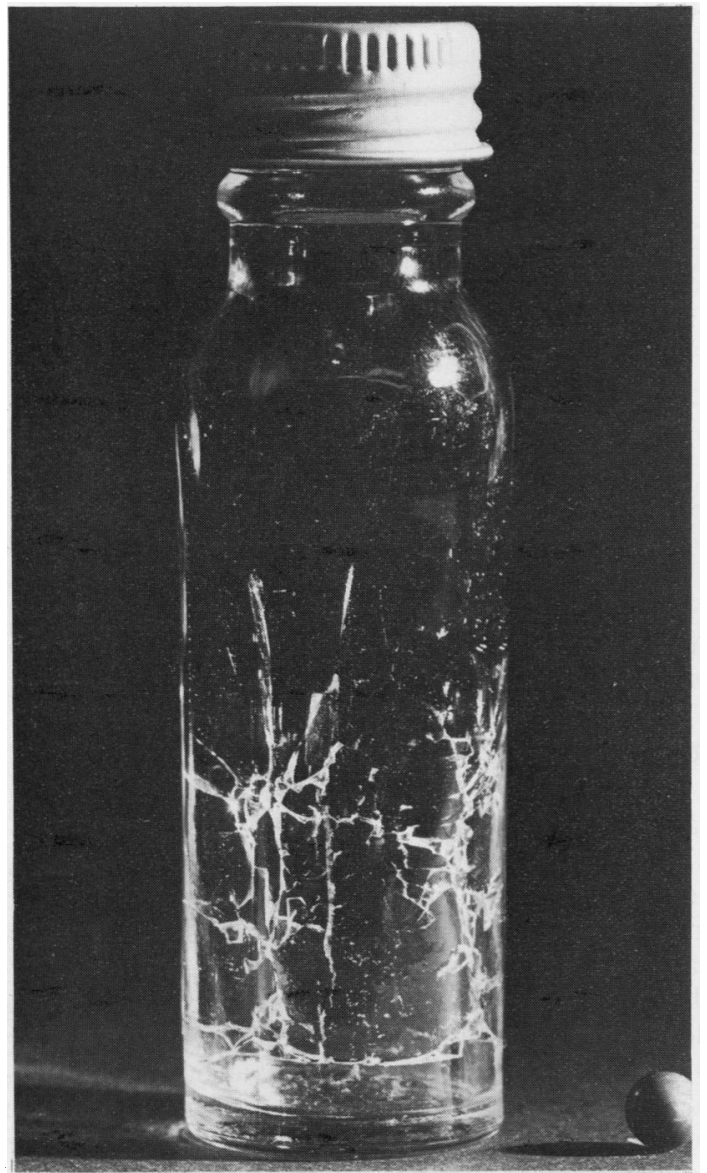

Fig. 1 Cracked bottle and ball bearing.

tamination with the test micro-organism. Decontamination of the test chamber and contents with formaldehyde vapour was carried out after each test and the chamber was thoroughly aired by means of an extract fan.

Three series of tests were performed.

\section{(a) Preliminary tests}

Before testing production models of sealed containers some preliminary work was carried out to prove the method and to assess potential hazards. This was done in a general purpose bench centrifuge using both swing out and angle rotors. Open mouth intact bottles were centrifuged to measure any aerosol generation in the absence of breakage. The result of breakage in screw capped bottles held in open buckets and the protection afforded by sealed buckets were assessed. Aerosol generation arising from a

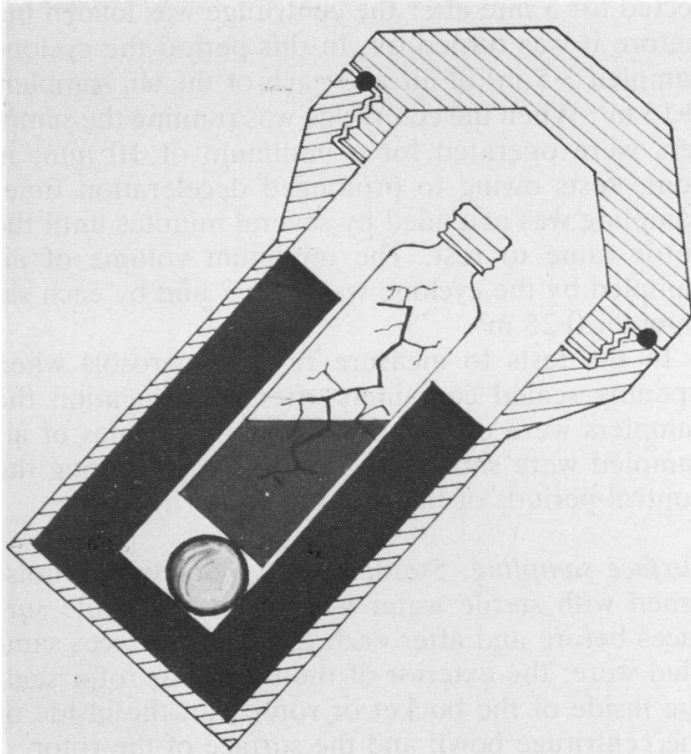

Fig. 2 General arrangement of test bucket showing position of ball bearing.

contaminated rotor was also measured. The effect of full application of the brake was compared with allowing the rotor to come to rest without any assistance.

(b) Tests of production models

Nineteen models of sealed buckets and seven sealed rotors were tested using cracked bottles.

(c) Residual aerosols in sealed buckets and rotors On completion of tests using production models of sealed buckets the residual aerosols present within the containers were measured on some occasions by sampling the air inside the test cabinet when the containers were opened several minutes after the centrifuge rotor had come to rest.

\section{SAMPLING METHODS}

Air sampling

A large volume glass cyclone, similar to that described by Decker et $a l,{ }^{4}$ and two slit samplers were run simultaneously throughout each test. The air inlets of the samplers were placed close to the centrifuge. For the cyclone this was about $30 \mathrm{~cm}$ from and about $10 \mathrm{~cm}$ above the lid. Slit sampler inlets were on opposite sides of the centrifuge level with the junction of the centrifuge lid and body at a distance of about $10 \mathrm{~cm}$.

\section{Air sampling programme}

For test series (a) and (b) control samples were col- 
lected for $5 \mathrm{~min}$ after the centrifuge was loaded but before it was turned on. In this period the cyclone sampled $3.5 \mathrm{~m}^{3}$ of air and each of the slit samplers $0.13 \mathrm{~m}^{3}$. When the centrifuge was running the samplers were operated for a minimum of $10 \mathrm{~min}$; in some tests owing to prolonged deceleration times sampling was extended by several minutes until the rotor came to rest. The minimum volume of air sampled by the cyclone was $7 \cdot 0 \mathrm{~m}^{3}$ and by each slit sampler $0.25 \mathrm{~m}^{3}$.

In the tests to measure residual aerosols when opening sealed containers after centrifugation the samplers were operated for $5 \mathrm{~min}$. Volumes of air sampled were similar to those collected during the control periods of the series (a) and (b) tests.

Surface sampling. Sterile cotton wool swabs moistened with sterile water were used to sample surfaces before and after each test. The surfaces sampled were: the exterior of the bucket or rotor seal; the inside of the bucket or rotor cap; the inside of the centrifuge bowl; and the surface of the rotor.

Assessment of samples. All cultures were made on the surface of plates of tryptose soya broth agar incubated before use to check sterility. Cyclone samples and suspensions, diluted where necessary in sterile phosphate buffer, were inoculated in measured volumes on to the surface of at least four plates. Swabs were rubbed over the surface of similar plates. Slit sampler plates were incubated without further processing. After incubation at $37^{\circ} \mathrm{C}$ for 18 to $24 \mathrm{~h}$ colony counts were recorded as colony forming units (CFU).

Definition of a satisfactory bucket or rotor. If on three separate occasions the numbers of spores recovered by air sampling or swabbing during or after centrifuging did not exceed the numbers recovered from control samples collected before a test the container was considered satisfactory. In most tests no spores were recovered from any of the control samples. In practice, results were clear cut. When a container leaked the numbers of spores recovered during centrifugation were always considerably higher than any present in a control sample.

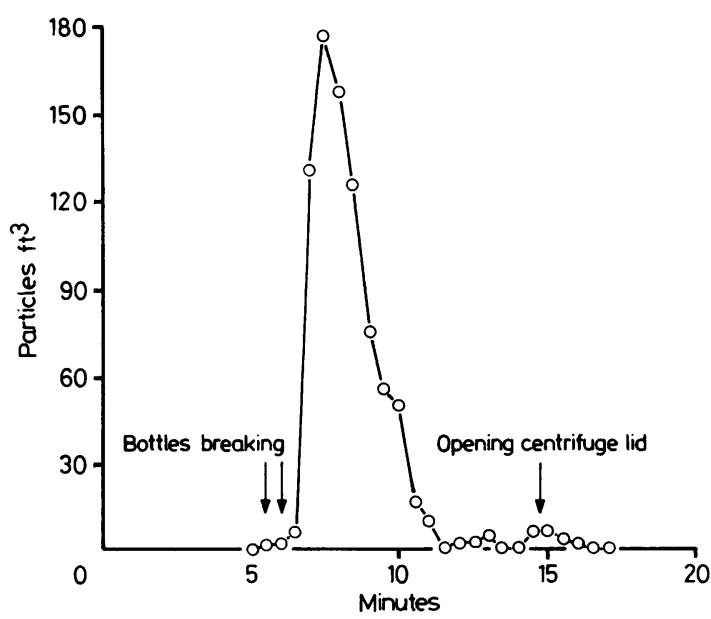

Fig. 3 Decay rate of airborne particles resulting from bottle breakage during centrifugation.

\section{Results}

\section{(a) Preliminary tests}

Table 1 shows that no aerosol was generated during centrifugation in open bottles which remained intact. Breakage of two bottles closed with screw caps generated about $1 \times 10^{4}$ particles. When bottles broke inside sealed buckets no aerosol was detected. Deliberate contamination of the rotor with $3 \times 10^{8}$ spores resulted in heavy airborne contamination. These findings agree with the work of others. $^{56}$ Fig. 3 shows the results from slit samplers collected when two bottles closed with screw caps broke during centrifugation. Application of the centrifuge brake did not result in the generation of airborne particles from open mouthed bottles.

\section{(b) Tests with production models of sealed contain- ers}

Results of these test are summarised in Table 2 . Of the items tested $27 \%$ failed to meet the criterion for satisfactory containment of aerosols generated as an outcome of bottles breaking during centrifugation. The reasons for some of these failures are discussed below.

Table 1 Aerosol recoveries by a cyclone sampler during centrifugation

\begin{tabular}{|c|c|c|c|c|c|}
\hline Rotor & Buckets & Bottles & State & $C F U$ in sample & Peak concentration $\left(f t^{3}\right)$ \\
\hline $\begin{array}{l}\text { Angle } \\
\text { Swing out } \\
\text { Swing out } \\
\text { Swing out } \\
\text { Swing out (contaminated) } \dagger\end{array}$ & $\begin{array}{l}\text { Open } \\
\text { Open } \\
\text { Open } \\
\text { Sealed } \\
\text { Open }\end{array}$ & $\begin{array}{l}\text { Open } \\
\text { Open } \\
\text { Closed } \\
\text { Closed } \\
\text { Open }\end{array}$ & $\begin{array}{l}\text { Intact } \\
\text { Intact } \\
\text { Broken } \\
\text { Broken } \\
\text { Intact }\end{array}$ & $\begin{array}{l}<5,<5^{*} \\
<5,<5,<5,<5^{*},<5^{*} \\
9.9 \times 10^{3} \\
<5,<5 \\
2.4 \times 10^{5}, 1.4 \times 10^{5}\end{array}$ & $\begin{array}{l}<0.004 \\
<0.004 \\
1.8 \times 10^{2} \\
<0.004 \\
>7.5 \times 10^{2}\end{array}$ \\
\hline
\end{tabular}

*Full application of rotor brake.

†Rotor contaminated with $9 \times 5 \mu$ l drops: total spores $=3 \times 10^{8}$. 
Table 2 Summary of tests on sealed buckets and rotors for aerosol leakage using a dynamic microbiological method

\begin{tabular}{|c|c|c|c|c|}
\hline Test items & $\begin{array}{l}\text { No of items } \\
\text { tested }\end{array}$ & Passed & Failed & $\%$ failed \\
\hline $\begin{array}{l}\text { Sealed buckets } \\
\text { Sealed rotors } \\
\text { Total }\end{array}$ & $\begin{array}{r}19 \\
7 \\
26\end{array}$ & $\begin{array}{r}15 \\
4 \\
19\end{array}$ & $\begin{array}{l}4 \\
3 \\
7\end{array}$ & $\begin{array}{l}21 \\
43 \\
27\end{array}$ \\
\hline
\end{tabular}

Table 3 Recovery of spores in air samples collected during the opening of sealed centrifuge buckets containing broken bottles

\begin{tabular}{|c|c|c|c|c|c|}
\hline & $C F U i$ & cyclone sam & aple & & \\
\hline & $<10^{2}$ & $>10^{2}-<10^{3}$ & $>10^{3}-<10^{4}$ & $>10^{4}-<10^{5}$ & $>10^{5}$ \\
\hline No of samples & 22 & 22 & 12 & 4 & 1 \\
\hline
\end{tabular}

\section{(c) Residual aerosols in sealed containers}

On 61/65 occasions airborne particles were recovered by a cyclone sampler during the opening of sealed buckets in which glass bottles containing spores had broken during centrifugation. Table 3 shows that the numbers of $\mathrm{CFU}$ recovered ranged between $<10^{2}$ and $>10^{5}$.

\section{Discussion}

Preliminary tests reported here, and by other workers, ${ }^{56}$ have shown that airborne particles are generated when breakage of a container or spillage occurs during centrifugation. Containment of this hazard can be achieved by enclosing the centrifuge in specially adapted Class III microbiological safety cabinets. $^{7-10}$ These cabinets are large and expensive, however, and for clinical laboratories sealed buckets and rotors are more convenient. Although buckets and rotors purporting to contain aerosols have been available for many years, and their sealing does not call for anything other than simple engineering, it is disturbing to find that some of what are described as safe pieces of equipment fail to contain aerosols. It is of interest to look at some of the reasons for the high failure rate found in tests with production models of sealed buckets and rotors.

\section{SEALED BUCKETS}

One model (Fig. 4) did not have a sealed gasket between the bucket and cap and there was no means of securing the cap. Not surprisingly this bucket failed to contain liquid or aerosols when bottles broke during centrifugation (Fig. 5). Two models of non-circular buckets fitted with rubber gaskets between bucket and cap secured by pressure at two points on the long sides of the bucket failed to contain aerosols and one failed to contain liquid. The use of circular buckets with O-ring seals was usually

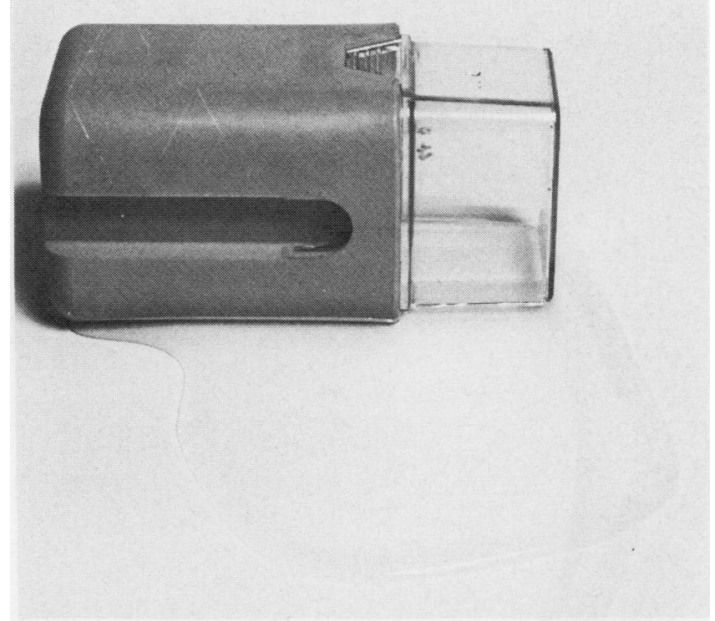

Fig. 4 Leakage of liquid from bucket without a gasket or cap retaining device.

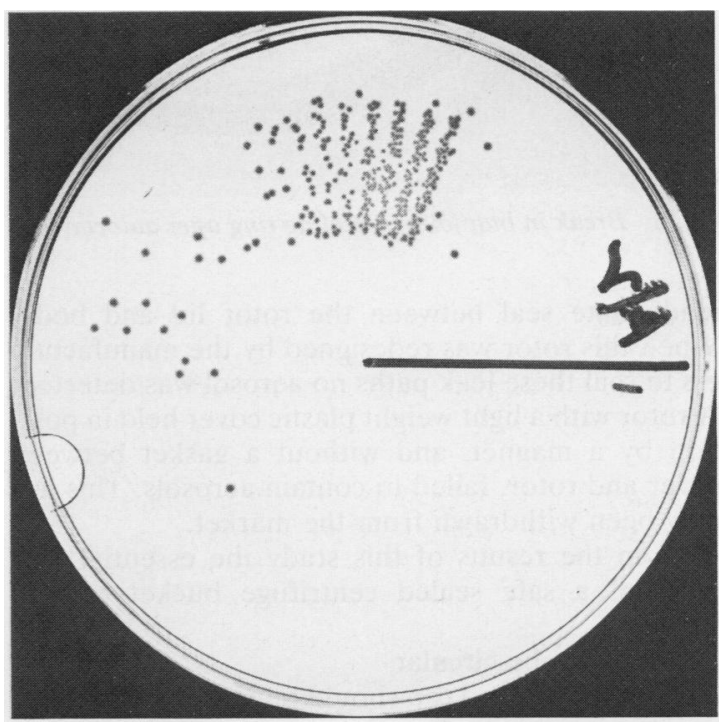

Fig. 5 Slit sampler plate showing the presence of particles escaping from an unsatisfactory sealed bucket.

satisfactory. It is essential, however, that only good quality moulded O-rings are used. One bucket with a seal made from a length of circular section rubber with a cemented butt joint failed owing to parting of the joint when it was autoclaved (Fig. 6).

\section{SEALED ROTORS}

Failure of one rotor was due to absence of seals between the lid latch and the body of the rotor, between the centrifuge spindle and the rotor, and an 


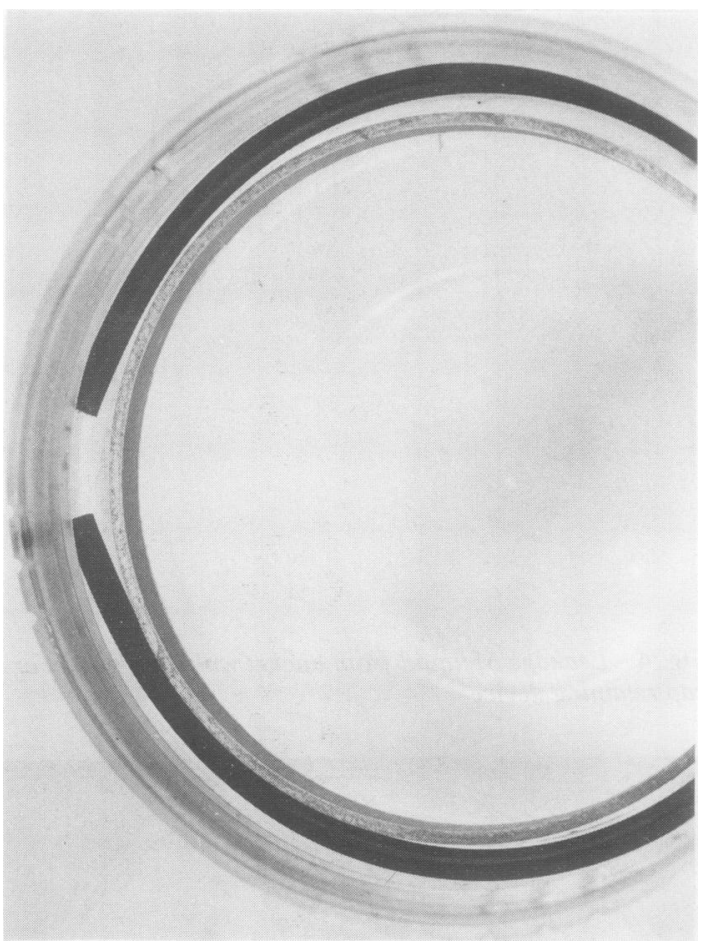

Fig. 6 Break in butt joint of sealing ring after autoclaving.

inadequate seal between the rotor lid and body. When this rotor was redesigned by the manufacturers to seal these leak paths no aerosol was detected. A rotor with a light weight plastic cover held in position by a magnet, and without a gasket between cover and rotor, failed to contain aerosols. This has now been withdrawn from the market.

From the results of this study the essential features of a safe sealed centrifuge bucket can be stated:

1 It should be circular

2 The bucket and cap should be made from metal; stainless steel is preferred

3 It should seal by all round pressure on a moulded O-ring seal recessed into the bucket

4 The sealing cap should screw into the bucket, not close over the outside of the bucket

5 The whole assembly of bucket, cap, and gasket should be able to withstand repeated sterilisation in an autoclave at $121^{\circ} \mathrm{C} .^{\prime}$

Clear plastic caps are sometimes fitted to sealed buckets so that the user can inspect the contents for damage before deciding whether they need to be opened in a safety cabinet. The use of these caps is to be discouraged for two reasons. Tubes or bottles which appear to be intact when viewed from above sometimes prove to be broken or to have discharged their contents when they are removed from the buckets. The findings of residual aerosols inside sealed buckets in which bottles have broken during centrifugation supports the requirement ${ }^{11}$ that sealed centrifuge containers must be opened inside a microbiological safety cabinet. A second reason is that on occasions, usually due to faulty loading of the centrifuge, buckets can become dislodged from the trunnions and in these circumstances the plastic caps can fracture inside the centrifuge bowl. The use of buckets and caps made from different materials has also led to problems in sterilising infected containers. Some of the plastic caps cannot be autoclaved, whereas the associated buckets made of aluminium are susceptible to chemical attack, especially by hypochlorites.

The use of sealed rotors to accommodate large numbers of samples in place of individual sealed buckets has some attraction in saving time in a laboratory needing to process a lot of samples in contained conditions. There are certain disadvantages in this. If one container breaks or leaks inside a sealed rotor the whole load, and in some models this may be as many as 96 units, must be regarded as contaminated. Removal of sealed rotors for opening in a safety cabinet can present problems. One rotor marketed for use in a bench top centrifuge weighs $13.6 \mathrm{~kg}(30 \mathrm{lb})$ when fully loaded. The removal of this bulky and heavy rotor calls for a considerable physical effort, and for safety it needs to be moved on a trolley. Another rotor, of continental manufacture, was too deep to pass through the open front of a standard Class I microbiological safety cabinet.

Although the dynamic method used in this work is slower than testing with fluorescein, and uses equipment not normally available in a clinical laboratory, it was used on the grounds of realism and sensitivity.

\section{References}

' British Standard 4402. Specification for safety requirements for laboratory centrifuges. London: British Standards Institution, 1982.

${ }^{2}$ Harper GJ. Microbiological safety tests. J Clin Pathol 1983;36: 117.

${ }^{3}$ Harper GJ. Contamination of the environment by special purpose centrifuges used in clinical laboratories. J Clin Pathol 1981;34:1114-23.

4 Decker HM, Buchanan WM, Frisque DE, Filler ME, Dahlgren CM. Advances in large-volume air sampling. Contamination Control 1969;8:13-7.

${ }^{5}$ Reitman M, Phillips GB. Biological hazards of common laboratory procedures. Am J Med Technol 1959;22:14-17.

- Kenny MT, Sable FL. Particle size distribution of Serratia marcescens aerosols created during common laboratory procedures and simulated accidents. Appl Microbiol 1968; 16: 1146-50. 
' Evans CGT, Harris-Smith R, Stratton JED. The use of safety cabinets for the prevention of laboratory acquired infections. In: Shapton DA, Board RG, eds. Safety in microbiology London and New York: Academic Press, 1972:21-36.

${ }^{8}$ Chatigny MA, Dun S, Ishimaru K, Eagleson JA, Prusiker SB. Evaluation of a Class III safety cabinet for enclosure of an ultra-centrifuge. Appl Environ Microbiol 1979;38:934-9.

${ }^{9}$ Gervin JL, Willis DD. Absolute containment of a preparative ultracentrifuge in hepatitis research. In: Proceedings of the National Cancer Institute symposium on centrifuge biohazards. Cancer Research Safety Monograph Series vol 1. Frederick, Maryland, USA: Litton Bionetics, 1973.

${ }^{10}$ Hencke CB. Containment and prototype equipment for ultracentrifuges in cancer research. In: Proceedings of the National
Cancer Institute symposium on centrifuge biohazards. Cancer Research Safety Monograph Series vol 1. Frederick, Maryland, USA: Litton Bionetics, 1973.

"Howie JW. A code of practice for the prevention of infection in clinical laboratories and post-mortem rooms (DHSS, working party). London: HMSO, 1978.

Requests for reprints to: Mr GJ Harper, Environmental Microbiology and Safety Reference Laboratory, Public Health Laboratory Service, Centre for Applied Microbiology and Research, Porton Down, Salisbury, Wiltshire, England. 